\title{
The BLUF-EAL protein YcgF acts as a direct anti-repressor in a blue-light response of Escherichia coli
}

\author{
Natalia Tschowri, Susan Busse, and Regine Hengge ${ }^{1}$ \\ Institut für Biologie-Mikrobiologie, Freie Universität Berlin, 14195 Berlin, Germany
}

The blue light using FAD (BLUF)-EAL protein YcgF is a known blue-light sensor of Escherichia coli, but its direct regulatory output and physiological function have remained unknown. Here, we demonstrate that unlike other EAL domain proteins, YcgF does not degrade the signaling molecule c-di-GMP, but directly binds to and releases the MerR-like repressor YcgE from its operator DNA upon blue-light irradiation. As a consequence, a distinct regulon of eight small proteins (of 71-126 amino acids) is strongly induced. These include YmgA and YmgB, which, via the RcsC/RcsD/RcsB two-component phosphorelay system, activate production of the biofilm matrix substance colanic acid as well as acid resistance genes and the biofilm-associated bdm gene and down-regulate adhesive curli fimbriae. Thus, small proteins under $\mathrm{YcgF} / \mathrm{YcgE}$ control seem to act as "connectors" that provide additional signal input into a two-component signaling pathway. Moreover, we found $y c g F$ and $y c g E$ expression to be strongly activated at low temperature, and we elucidate how blue light, cold, and starvation signals are integrated in the expression and activity of the $\mathrm{YcgF} / \mathrm{YcgE} / \mathrm{small}$ protein signaling pathway. In conclusion, this pathway may modulate biofilm formation via the two-component network when $E$. coli has to survive in an extrahost aquatic environment.

[Keywords: Biofilm; connectors; cyclic-di-GMP; GGDEF; RcsB; RpoS]

Supplemental material is available at http://www.genesdev.org.

Received July 25, 2008; revised version accepted January 5, 2009.

GGDEF and EAL domain proteins have been recognized recently to act as diguanylate cyclases (DGC) and phosphodiesterases (PDE), respectively, that "make and break" the ubiquitous bacterial signaling molecule bis- $\left(3^{\prime}, 5^{\prime}\right)$ cyclic-guanosine monophosphate (c-di-GMP). In general, c-di-GMP interferes with motility and promotes biofilm formation; i.e., it acts as a key signal in switching between the planktonic/single-cellular and sedentary/ multicellular "life-styles" (for recent reviews, see Jenal and Malone 2006; Römling and Amikam 2006; Ryan et al. 2006). In pathogenic bacteria, c-di-GMP can also regulate virulence genes (Cotter and Stibitz 2007; Tamayo et al. 2007). The GGDEF and EAL amino acid motifs are part of the respective active centers and therefore are essential for the activities of these enzymes (Malone et al. 2007; Wassmann et al. 2007; Rao et al. 2008). Many bacterial species possess up to several dozen GGDEF/EAL domain proteins. In these proteins, the two domains can occur either alone ("GGDEF-only" or "EAL-only") or in combination in single polypeptides ("GGDEF + EAL"). The majority of these proteins also carry $\mathrm{N}$-terminal sensory

${ }^{1}$ Corresponding author.

E-MAIL Rhenggea@zedat.fu-berlin.de; FAX 49-30-838-53118.

Article is online at http://www.genesdev.org/cgi/doi/10.1101/gad.499409. input domains that can control the activities of the GGDEF and/or EAL domains.

Intriguingly, in a certain fraction of these proteins, the GGDEF and/or EAL motifs as well as additional amino acids relevant for enzymatic function are not conserved. This is found not only in GGDEF + EAL proteins, where one domain can be degenerate and the other then determines the enzymatic activity, but also in proteins where no intact GGDEF or EAL domains remain. These proteins should not have DGC or PDE activities, but act in different ways. Among the 29 GGDEF/EAL proteins of Escherichia coli, there are four such proteins: YeaI (a GGDEF-only protein), YhdA (featuring GGDEF and EAL domains, but both degenerate), and the two EAL-only proteins, YcgF and YdiV. The functions of YeaI, YcgF, and YdiV have remained unknown. YhdA promotes the turnover of two small RNAs, CsrB and CsrC, which by sequestering the RNA-binding protein CsrA can reduce the expression of the flagellar master regulator FlhDC at the post-transcriptional level (Suzuki et al. 2006).

In the present study, we unravel the molecular and physiological function of the YcgF protein, in which the degenerate EAL domain is preceeded by an $\mathrm{N}$-terminal blue-light-sensing BLUF domain ("blue light using FAD") (Gomelsky and Klug 2002; Purcell and Crosson 2008). In 
biophysical studies, conformational changes upon bluelight irradiation have been observed in the BLUF domain as well as in the entire YcgF protein, and the protein is believed to dimerize upon photo-excitation (Rajagopal et al. 2004; Hasegawa et al. 2006; Nakasone et al. 2007; Schroeder et al. 2008). However, the molecular output function controlled by the BLUF domain as well as the physiological context, where all this plays a role for $E$. coli, have remained elusive.

Here, we demonstrate that YcgF does not have PDE activity, but in a process that requires its EAL domain, it is a directly interacting antagonist for a repressor, YcgE. Blue-light-induced YcgF-mediated release of YcgE from its target operators results in the expression of a distinct set of small regulatory proteins, which, via the Rcs phosphorelay system, affects the production of colanic acid-i.e., a biofilm matrix component-and adhesive curli fimbriae. Moreover, we show that expression of $\mathrm{YcgF}$ and $\mathrm{YcgE}$ as well as their target genes are strongly induced by low temperature. Thus, YcgF is the first EAL protein shown to act by direct protein-protein interaction, and in doing so, in response to blue light, it acts as the sensory input component of a novel signal transduction pathway of a complex blue light and cold stress response that controls biofilm formation in an extrahost environment.

\section{Results}

Identification of target genes of the MerR-like regulator YcgE

On the E. coli chromosome, the $y c g F$ gene is followed by ycgE, which encodes a MerR-like regulator of unknown function. Whether the two genes constitute an operon was not clear at the beginning of this study (see Materials and Methods for further details). What drew our attention to YcgE was the finding that not only does it contain a MerR-like N-terminal domain, but also that the complete protein shows clear homology with MlrA, which is an activator essential for the expression of the curli regulator CsgD (Brown et al. 2001). csgD transcription is also regulated by YdaM and YciR; i.e., a DGC (GGDEFonly) and a PDE (with GGDEF and EAL domains). In other words, here was a precedent for a MerR-like protein and an EAL protein cooperating in transcription initiation (Weber et al. 2006). We therefore suspected that YcgE and YcgF might act in a common pathway that controls the expression of an unknown set of genes.

As a first step, we identified YcgE-regulated genes, as this would allow us to develop a convenient reporter assay for the function of $\mathrm{YcgE}$ and, possibly, YcgF. Genome-wide transcriptional profiling on microarrays revealed a small set of eight genes that are derepressed in a $y c g E$ knockout mutant (in cells grown in $\mathrm{LB}$ at $28^{\circ} \mathrm{C}$ ) (Supplemental Table S1): Whereas two major acid resistance genes (gadB, hdeB) showed only moderate derepression (threefold to fourfold), six other genes of unknown molecular functions $(y c g Z, y l i L, y m g A, y m g B$, $y m g C$, and ynaK) were rather strongly activated in the ycgE mutant (sixfold to 22 -fold).

This latter group of genes is remarkable in several aspects. First, all six genes encode small proteins of 78-90 amino acids with unknown molecular functions (see also in Table 1). Second, $y c g Z, y m g A$, and $y m g B$ are under the control of the general stress $\sigma$ factor $\sigma^{\mathrm{S}}$ (RpoS) (Weber et al. 2005), and their arrangement on the E. coli chromosome suggested that they might constitute an operon. $y m g C$ is the next gene downstream from this region, but it did not show significant control by $\sigma^{\mathrm{S}}$ (Weber et al. 2005). However, Northern blot analysis with probes complementary to $y c g Z$ and $y m g C$ indicated that all four genes are expressed in a single polycistronic mRNA of $\sim 1400$ nucleotides (nt) (our unpublished results; see also

Table 1. Genes differentially expressed in $M C 4100\left(y c g F^{+}\right)$and its otherwise isogenic ycgF::kan derivative as determined by genome-wide transcriptional profiling on microarrays ${ }^{a}$

\begin{tabular}{|c|c|c|c|c|c|c|}
\hline \multirow[b]{2}{*}{ Gene $^{b}$} & \multirow[b]{2}{*}{ b-number } & \multirow{2}{*}{$\begin{array}{c}\text { Protein size } \\
\text { (amino acids/kilodalton) }\end{array}$} & \multirow{2}{*}{$\begin{array}{l}\text { YcgF dependence } \\
\left(\text { ratio: } y c g F^{+} / \text {ycgF }\right)\end{array}$} & \multicolumn{3}{|c|}{$\begin{array}{c}\sigma^{\mathrm{S}} \text { dependence } \\
\left(\text { ratio: }^{\prime} \text { rpoS }^{+} / \text {rpoS }^{-}\right)^{\mathrm{c}}\end{array}$} \\
\hline & & & & LB/OD4 & $\mathrm{NaCl}$ & pH 5 \\
\hline ybgs & b0753 & $126 / 12.8$ & 3.68 & 8.43 & 38.08 & 16.12 \\
\hline yliL & b0816 & $89 / 10.0$ & 40.56 & - & - & - \\
\hline ycgZ & b1164 & 78/8.7 & 12.66 & 5.11 & 5.53 & 4.00 \\
\hline$y m g A$ & b1165 & $90 / 10.3$ & 60.20 & 4.98 & 5.89 & 4.63 \\
\hline$y m g B$ & b1166 & $88 / 9.6$ & 85.90 & 3.30 & 3.84 & - \\
\hline$y m g C$ & b1167 & $82 / 9.7$ & 70.70 & - & - & - \\
\hline ynaK & b1365 & $87 / 9.7$ & 27.74 & - & - & - \\
\hline$b d m(y d d X)$ & b1481 & $71 / 7.9$ & 8.66 & 2.73 & 4.64 & 4.10 \\
\hline
\end{tabular}

a The two strains were grown in LB medium at $37^{\circ} \mathrm{C}$ to an $\mathrm{OD}_{578}$ of 0.7 , then shifted to $16^{\circ} \mathrm{C}$ and exposed to blue light for three additional hours before samples for RNA preparation were taken. For details of microarray analysis, see the Materials and Methods. ${ }^{\mathrm{b}}$ Genes are ordered according to b-numbers (reflecting chromosomal position).

${ }^{\mathrm{c}}$ Data were taken from Weber et al. (2005), where $r p o S^{+} / r p o S^{-}$ratios were determined under three different conditions that result in the

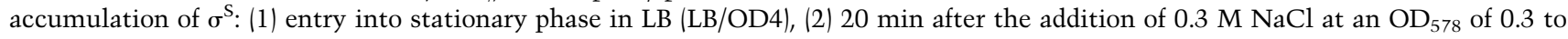
cultures growing in $\mathrm{M} 9 / \mathrm{glycerol}(\mathrm{NaCl})$, and (3) $40 \mathrm{~min}$ after a shift to $\mathrm{pH} 5$ at an $\mathrm{OD}_{578}$ of 0.4 of cultures growing in $\mathrm{LB}$ (pH 5). (-) Ratio is $<2.00$; i.e., no significant $\sigma^{\mathrm{S}}$ dependence. The full $\mathrm{rpoS}^{+/-}$data sets can also be found on http://www.biologie.fu-berlin.de/en/microbio/ mibi-hengge/scidata/index.html. 
Fig. 5, below). ymgC may have an additional operoninternal and $\sigma^{\mathrm{S}}$-independent start site, whose activity could reduce the relative influence of $\sigma^{\mathrm{s}}$ in ymgC expression. Strikingly, the $y c g Z-y m g A-y m g B-y m g C$ region is located right next to the $y c g F-y c g E$ region, with a divergently transcribing control region in between, consistent with all these genes acting in a common regulatory and functional context. yliL and ynaK are located in other regions of the E. coli chromosome, indicating that YcgE is a global rather than a local regulator.

\section{The BLUF-EAL protein YcgF acts in a regulatory pathway upstream of $Y c g E$}

In order to study the regulatory roles of $\mathrm{YcgE}$ and $\mathrm{YcgF}$, a single-copy ycgZ::lac $Z$ reporter fusion was constructed [containing the entire intergenic control region present upstream and integrated at $a t(\lambda)$ in the chromosome]. In an otherwise wild-type background, this reporter fusion showed weak expression that was slightly stimulated during entry into stationary phase (Fig. 1A). Consistent with microarray data (Supplemental Table S1; Weber et al. 2005), ycgZ expression was strongly derepressed in the $y c g E$ mutant and positively $\sigma^{\mathrm{S}}$-regulated both in $y c g E^{+}$and $y c g E$ mutant strains (Fig. 1A); in the $y c g E$ mutant, eliminating $\sigma^{\mathrm{S}}$ only partially reduced expression, suggesting that the single $y c g Z$ promoter can also be activated to some extent by vegetative RNA polymerase in the absence of $\sigma^{\mathrm{S}}$ (our unpublished data). In the $y c g F$ mutant, $y c g Z$ expression was reduced (Fig. 1B). In the absence of YcgE, however, the $y c g F$ mutation no longer affected $y c g Z$ expression, which was similarly elevated as in the mutant defective in $y c g E$ alone (Fig. 1A,B). These data indicate that $\mathrm{YcgF}$ plays an activating role in the control of $y c g Z$ and that it acts upstream of YcgE in a common pathway. This was further confirmed with a YcgF-overexpressing plasmid, which activated ycgZ expression to the same extent as the mutation in $y c g E$, and this effect was not additive to that of the $y c g E$ mutation (Fig. 1C). Taken together, these data suggest that the BLUF-EAL protein YcgF is a direct or indirect antagonist for the transcriptional regulator YcgE.

\section{YcgF does not have phosphodiesterase activity, but} acts as a direct antagonist for $\mathrm{Ycg} E$

How does YcgF antagonize the action of YcgE? In the degenerate EAL domain of $\mathrm{YcgF}$, all four amino acids required to bind c-di-GMP and three amino acids crucial for $\mathrm{Mg}^{2+}$ binding and catalysis (Rao et al. 2008) are not conserved (Supplemental Fig. S1), thus making it highly unlikely that YcgF acts via c-di-GMP. Nevertheless, we wanted to clarify whether the EAL domain of YcgF may still have some PDE activity. We purified both YcgF as well as a mutated protein in which the degenerate motif EAIVQ was replaced by the consensus signature EALVR ${ }^{(Y c g F}{ }^{1193 L+Q 195 R}$; this protein was as efficient as the wildtype protein in activating ycgZ::1acZ expression, when expressed from a plasmid) (data not shown). In contrast to a positive control (YhjH, a known PDE) (Pesavento et al. 2008), neither YcgF variant cleaved c-di-GMP, no matter whether blue-light-irradiated or not (Supplemental Fig. S2A). Thus, the degenerate EAL domain of YcgF does not exhibit PDE activity, and also "repairing" just the EAL

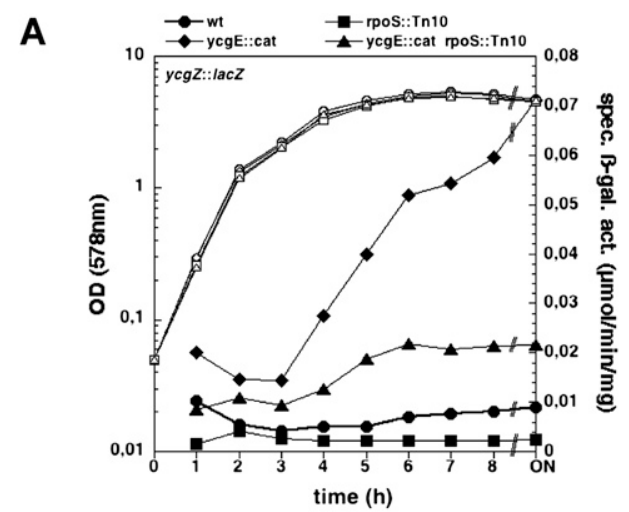

B
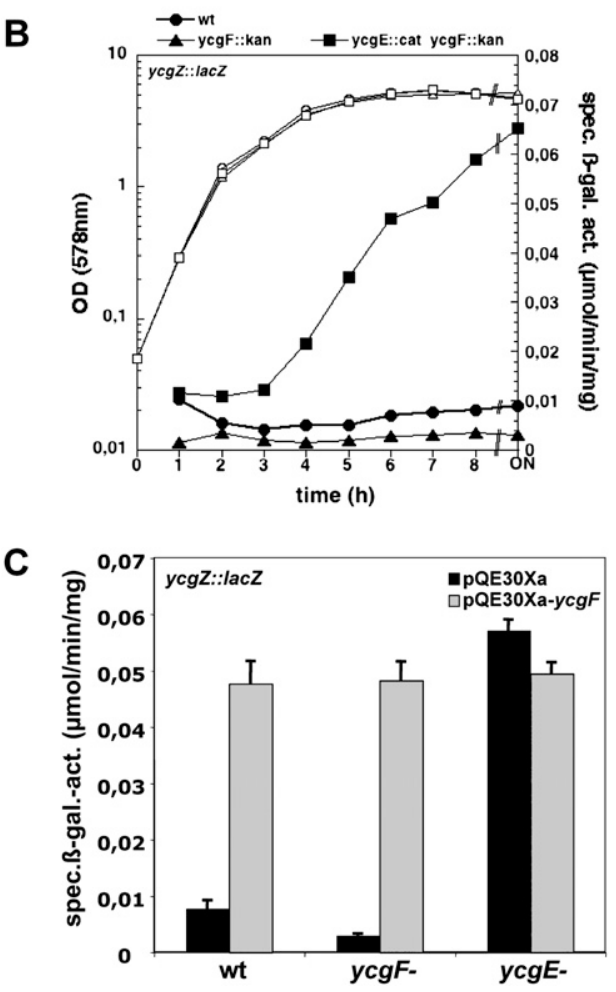

Figure 1. YcgE is a repressor for $y c g Z$ expression and is antagonized by YcgF. $(A)$ ycgZ expression is $\sigma^{\mathrm{S}}$-dependent and derepressed in a $y c g E$ mutant. Strain MC4100 containing a singlecopy ycgZ ::lacZ reporter fusion (wt) and its derivatives carrying mutations in rpoS and ycgE either alone or in combination as indicated were grown in $\mathrm{LB}$ at $37^{\circ} \mathrm{C}$. $\mathrm{OD}_{578}$ (open symbols) and specific $\beta$-galactosidase activities (closed symbols) were determined along the growth curve. $(B)$ A ycgF mutation reduces $y c g Z$ expression in the presence but not in the absence of YcgE. Derivatives of MC4100 carrying ycgZ::1acZ and $y c g E$ and $y c g F$ mutations as indicated were grown and analyzed as described for A. (C) A ycgF-carrying plasmid activates ycgZ expression, provided the repressor YcgE is present. Derivatives of MC4100 carrying $y c g Z:: 1 a c Z$ as well as mutations in $y c g F$ and $y c g E$ as indicated were transformed with a YcgF-overproducing plasmid (pQE30Xa$y c g F)$ or the vector alone (pQE30Xa), and $\beta$-galactosidase activities were determined after $24 \mathrm{~h}$ growth in $\mathrm{LB} /$ ampicilline at $37^{\circ} \mathrm{C}$. 
motif alone is not sufficient to restore such activity, as several important amino acids at other key positions of the EAL domain are not present (Supplemental Fig. S1). In addition, both wild-type YcgF as well as the "improved EAL" variant were also unable to bind c-di-GMP (Supplemental Fig. S2B). These data indicate that c-di-GMP is not involved in the activity of YcgF. Moreover, we observed that overexpression of the DGC YaiC or the PDE YhjH did not alter the expression of the target gene $y c g Z$ (Supplemental Fig. S3; note that overexpression of YaiC and $\mathrm{YhjH}$ did have the expected effects on motility as detailed in the figure legend). In addition, knockout mutations in all genes encoding proteins with intact GGDEF motifs (i.e., known or putative DGCs) did not affect $y c g Z::$ lac $Z$ expression (data not shown). Altogether, this means that despite YcgF being an EAL protein, c-diGMP does not play a role in the $\mathrm{YcgF} / \mathrm{YcgE}$ regulatory pathway.

We therefore tested whether YcgF and YcgE interact directly. Full-size YcgE as well as its N-terminal MerRlike domain (YcgE-NTD) and the C-terminal domain (YcgE-CTD) were cloned and purified with a TRXHis6-S tag at their N-terminal ends, which allows specific binding to S-protein agarose. In affinity chromatography ("pull-down") experiments, putative interaction of these proteins with purified full-size YcgF and the YcgF-BLUF domain alone was tested (the C-terminal YcgF-EAL domain alone was extremely prone to aggregation and could not be isolated in soluble form). Not only did the full-size proteins YcgE and YcgF interact (Fig. 2A), but specifically the N-terminal MerR-like domain of YcgE was able to bind to YcgF (Fig. 2B). As no interaction was observed with the N-terminal YcgF-BLUF domain alone, we conclude that the C-terminal EAL domain of YcgF (either alone or in cooperation with the BLUF domain) is essential for this direct interaction with the MerR-like YcgE-NTD. The "improved EAL" variant of YcgF also bound to YcgE as well as to YcgE-NTD (data not shown).

This direct interaction suggested that YcgF may modulate the activity of the MerR-like domain of YcgE; i.e., DNA binding. We therefore tested YcgE binding to the $y c g Z$ promoter region $\left(y c g Z_{\mathrm{p}}\right)$ and a potential effect of $Y c g F$ thereon in electrophoretic mobility shift assays. YcgE was found to bind specifically to the $y c g Z_{\mathrm{p}}$ DNA fragment; i.e., YcgE plays a direct role in ycgZ transcriptional repression (Fig. 3; Supplemental Fig. S4). YcgF, in contrast, did not show any binding, when added alone to the $y c g Z_{\mathrm{p}}$ DNA fragment (Fig. 3). When YcgF and YcgE were both present in the assay, YcgE was released from the DNA in a YcgF dose-dependent manner (Fig. 3A). This release was dependent on blue-light irradiation and was modulated by the order of addition of the relevant components. When $Y c g E$ and $y c g Z_{\mathrm{p}}$ DNA were preincubated before the addition of YcgF and blue-light irradiation (Fig. 3A), more YcgF was required to chase YcgE from the DNA than when YcgE and YcgF were preincubated and blue-light-irradiated before the addition of DNA (Fig. $3 \mathrm{~B}$ ), and nearly stoichiometric amounts of YcgF were sufficient to completely release YcgE, when the latter

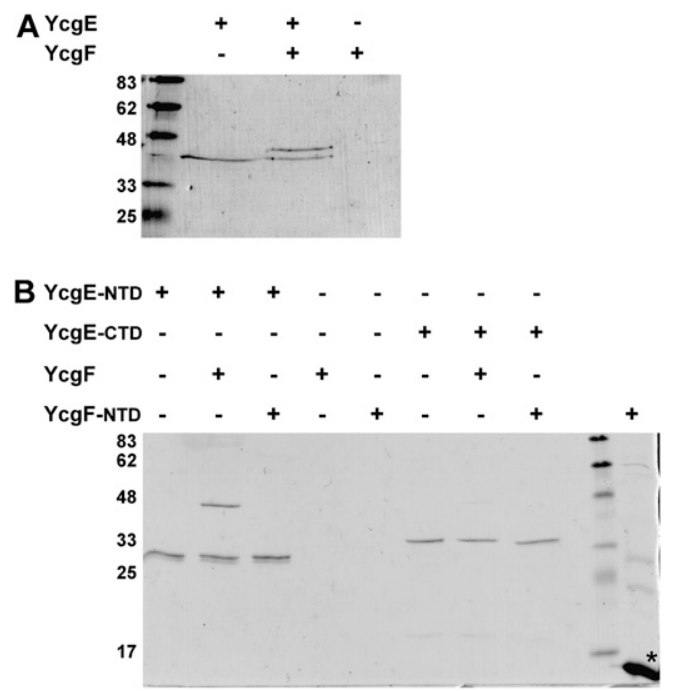

Figure 2. The N-terminal MerR-like domain of YcgE directly interacts with $\mathrm{YcgF}$ in vitro. $\mathrm{YcgE}$ and $\mathrm{YcgF}(A)$ as well as their isolated domains as indicated $(B)$ were purified (YcgE-NTD is the MerR-like domain of YcgE; YcgF-NTD is the BLUF domain of YcgF; see also Fig. 7). These proteins were used for affinity chromatography ("pull-down") and coelution experiments on Sprotein agarose, to which YcgE, YcgE-NTD, and YcgE-CTD bind via their TRX-His6-S tag (for details, see the Materials and Methods). The last lane in $B$ contains purified YcgF-NTD (i.e., the BLUF domain) for comparison, as this protein does not coelute in any other lane.

procedure was further modified to include a second irradiation after DNA addition (Fig. 3C). These data suggest high affinities and low off-rates of the interaction reactions involved. This is also consistent with no rebinding of $Y c g E$ to $y c g Z_{\mathrm{p}}$ DNA, when samples treated as in Figure 3A were kept in the dark for 60 min before running them on the gel (data not shown), and the slow reversion to the ground state in the photocycle of YcgF (Schroeder et al. 2008). In the absence of blue-light irradiation, even a relatively large excess of YcgF did not release YcgE from its operator. This means either that YcgF, despite possible binding to DNA-bound YcgE, does not induce a conformational change in YcgE required for its release from the operator site, or that YcgF does not efficiently bind to DNA-bound YcgE. The latter would be in contrast to YcgF binding to free YcgE in the absence of DNA /as in the interaction assay shown in Fig. 2), suggesting that $\mathrm{YcgF}$ and $y c g Z_{\mathrm{p}}$ DNA compete for overlapping binding sites on the YcgE MerR-like domain.

We conclude that blue-light irradiation, which is sensed by its BLUF domain and transmitted by conformational changes (Hasegawa et al. 2006; Nakasone et al. 2007), enables YcgF to interact with operator-bound YcgE and to release it from the DNA. Moreover, this biochemical activity of $\mathrm{YcgF}$ is fully consistent with the genetically defined role of $\mathrm{YcgF}$ as an antagonist to the YcgE repressor in the control of $y c g Z$ expression (Fig. 1). 


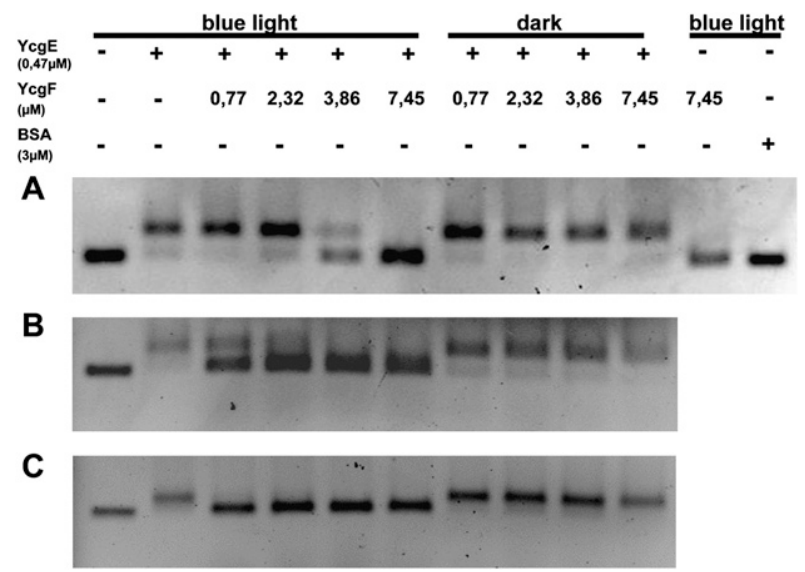

Figure 3. YcgF releases YcgE bound to its operator site in the $y c g Z$ control region in a blue-light irradiation-dependent manner. A 407-bp fragment carrying the $y c g Z$ promoter region together with purified YcgE and/or YcgF in the concentrations indicated was either blue-light-irradiated or kept in the dark for $20 \mathrm{~min}$ and analyzed by electrophoretic mobility shift assay. $(A)$ $\mathrm{YcgE}$ and $y c g Z_{\mathrm{p}}$ DNA were preincubated for $20 \mathrm{~min}$, before YcgF was added and the sample was subject to blue-light irradiation. (B) YcgE and YcgF were mixed, and the sample was blue-light irradiated before $y c g Z_{\mathrm{p}}$ DNA was added. $(C)$ The procedure was the same as in $B$, except for a second 20-min blue-light irradiation period after DNA addition.

The blue-light response initiated by the $Y c g F / Y c g E$ pathway is important at low temperature

The $\mathrm{YcgF} / \mathrm{YcgE}$ pathway responds to blue light as an input signal. Why and under what conditions is blue-light sensing relevant for $E$. coli? Is blue light just an indicator for being in a certain environment, or does blue-light irradiation itself cause stress; i.e., can it be detrimental to cellular physiology? It is obvious that any blue-light effects should occur in an outside environment rather than inside a mammalian host. Consistently, in a study of expression patterns of all GGDEF/EAL genes in E. coli (Sommerfeldt et al. 2009), it was observed that $y c g F$ belongs to a group of GGDEF/EAL genes that are more strongly expressed at $28^{\circ} \mathrm{C}$ than at $37^{\circ} \mathrm{C}$. Moreover, ycgF and $y c g Z$ are members of an E. coli cold shock stimulon described by two microarray studies (Polissi et al. 2003; White-Ziegler et al. 2008). This prompted us to study the regulation of the entire $\mathrm{YcgF} / \mathrm{YcgE} / \mathrm{ycgZ}$ system in closer detail (using single-copy lac $Z$ reporter fusions also to $y c g F$ and $y \operatorname{cg} E$; note that each of these two genes is expressed from its own transcriptional start site) (for details, see the Materials and Methods). We found that induction of $y c g F$ was even stronger when the temperature was reduced further below $28^{\circ} \mathrm{C}$, both after steady-state growth (Fig. $4 \mathrm{~A}$ ) as well as after a shift from $37^{\circ} \mathrm{C}$ to $16^{\circ} \mathrm{C}$ (Fig. 4B). $y c g E$, too, was increasingly expressed at lower temperature (Fig. 4A,C), although not as strongly as $y \operatorname{cgF}$ (the difference in expression at $16^{\circ} \mathrm{C}$ vs. $37^{\circ} \mathrm{C}$ was about sevenfold for $y c g E$, but $>30$-fold for $y c g F)$. Thus, the $\mathrm{YcgF} / \mathrm{YcgE}$ pathway seems especially important in an outside environment at low temperatures that support only slow growth of mesophilic bacteria like E. coli.
Consistently, the target gene $y c g Z$ also was induced upon shift from $37^{\circ} \mathrm{C}$ to $16^{\circ} \mathrm{C}$, and even more so if the culture was also exposed to blue-light irradiation in addition to the temperature downshift (Fig. 5). Both the analysis of the $y c g Z:: 1 a c Z$ fusion (Fig. $5 \mathrm{~A}$ ) as well as complete transcript analysis by Northern blotting (Fig. $5 B$ ) showed that the expression of $y c g Z$ (and the entire $y c g Z$-ymg $A B C \mathrm{mRNA}$ ) is completely dependent on YcgF,

A

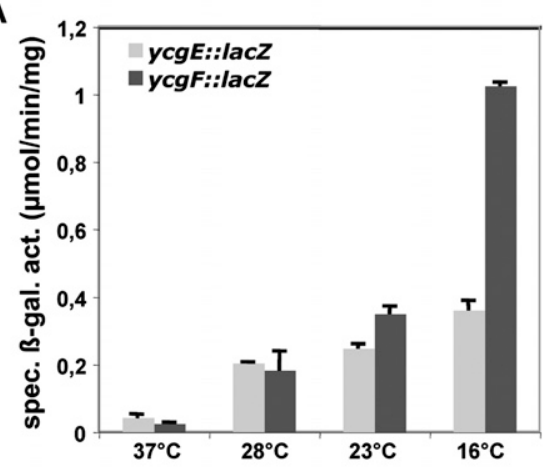

B

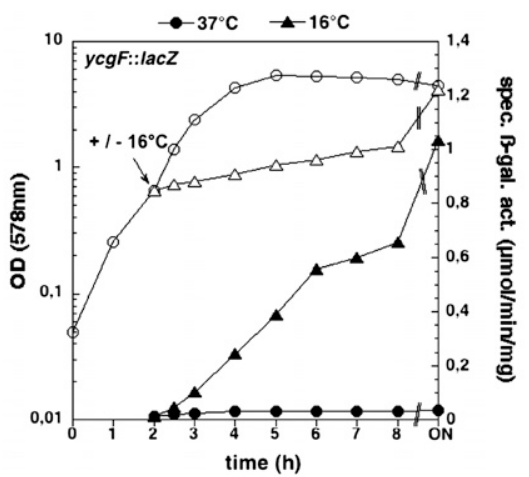

C

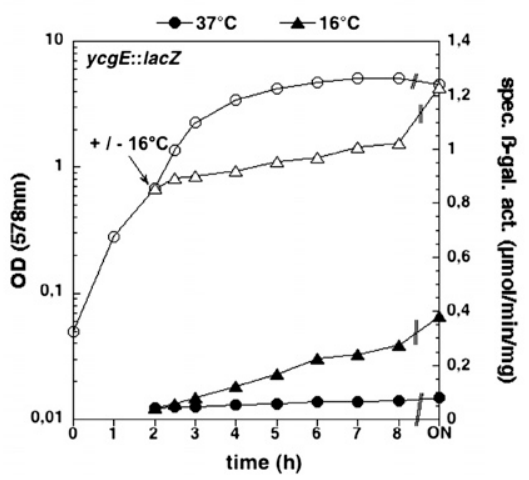

Figure 4. Expression of $y c g E$ and $y c g F$ is strongly activated by low temperature. Derivatives of strain MC4100 carrying singlecopy ycgE::lacZ and ycgF::lacZ reporter fusions were grown in LB. (A) Cultures were grown continuously at the temperatures indicated, and $\beta$-galactosidase activities were determined after growth for $24 \mathrm{~h}\left(37^{\circ} \mathrm{C}, 28^{\circ} \mathrm{C}\right.$, and $\left.23^{\circ} \mathrm{C}\right)$ or $66 \mathrm{~h}\left(16^{\circ} \mathrm{C}\right) .(B)$ MC4100 carrying ycgF::1acZ was grown at $37^{\circ} \mathrm{C}$ to an $\mathrm{OD}_{578}$ of 0.7 , where the culture was split and one of the two aliquots was shifted to $16^{\circ} \mathrm{C}$. $\mathrm{OD}_{578}$ (open symbols) and specific $\beta$-galactosidase activities (closed symbols) were determined along the growth curve. $(C)$ MC4100 carrying ycgE::lacZ was grown and analyzed as described for $B$. 
A

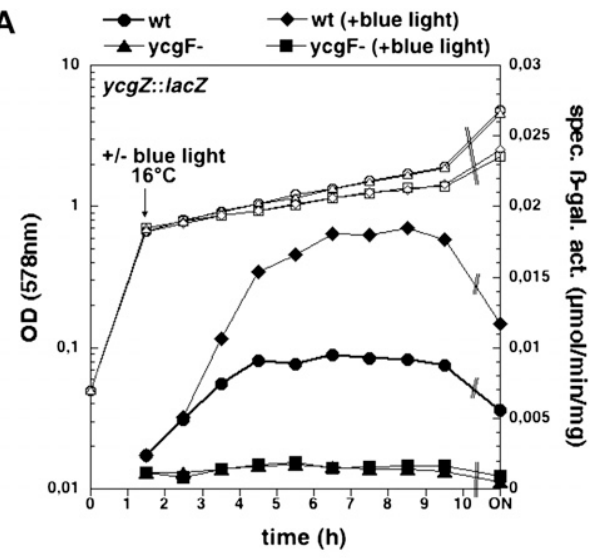

B

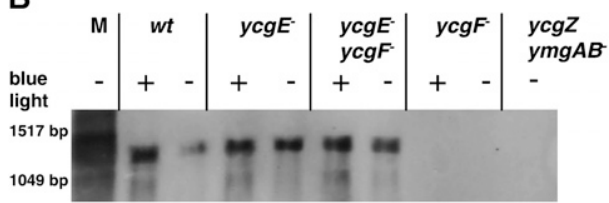

Figure 5. Blue light and temperature down-shift additively induce $y c g Z$ expression in a YcgF-dependent manner. $(A)$ MC4100 carrying $y c g Z:: 1 a c Z$ as well as its ycgF mutant derivative were grown in $\mathrm{LB}$ to an $\mathrm{OD}_{578}$ of 0.7 , where the cultures were shifted to $16^{\circ} \mathrm{C}$ and split into two aliquots that were either blue-light-irradiated or kept in the dark. $\mathrm{OD}_{578}$ (open symbols) and specific $\beta$-galactosidase activities (closed symbols) were determined along the growth curve. $(B)$ Expression of the ycgZ-ymgABC mRNA as detected by Northern blot analysis. MC4100 (wt) and its derivatives carrying ycgE::cat, ycgF::kan, or $\Delta(y c g Z$-ymg $A B):: c a t$ were grown as described for $A$. RNA was prepared $3 \mathrm{~h}$ after the shift to $16^{\circ} \mathrm{C}$ and subjected to Northern blot analysis using a probe corresponding to the $y c g Z$ coding region. The transcript detected has $\sim 1400 \mathrm{nt}$ and can also be detected with a probe corresponding to the $y m g C$ coding region (data not shown); i.e., it corresponds to the full-size operon transcript.

even when cells were exposed to $16^{\circ} \mathrm{C}$ only and not irradiated. Thus, also in the absence of blue light, YcgF can and is in fact required to relieve repression by YcgE at low temperatures (Fig. 5B).

Having learned that the $\mathrm{YcgF} / \mathrm{YcgE}$ system is particularly important in a cold environment, we wondered whether we had detected all target genes of YcgE in our initial microarray transcriptome experiments performed at $28^{\circ} \mathrm{C}$ (Supplemental Table S1). In addition, the question arose whether YcgF acts exclusively via YcgE or whether it may also have an additional effect on other regulatory pathways. We therefore compared the transcriptional profiles of $y c g F^{+}$and $y c g F$ mutant strains at $16^{\circ} \mathrm{C}$ and in the presence of blue light. The list of genes differentially affected was again small and nearly identical to that observed for $\mathrm{ycgE}^{+/-}$in the initial experiment (Table 1): Again, the entire $y c g Z$-ymgA-ymgB-ymgC operon as well yliL and ynaK were found. In addition, two new genes were detected, $b d m$ and $y b g S$, which also encode small proteins (71 and 126 amino acids, respectively) (Table 1). The two weakly YcgE-repressed acid resistance genes gadB and hdeB did not show significant differential expression on the $y c g F^{+/-}$microarrays under these conditions. Finally, we noted that the ratios of differential expression of the six genes found in both microarray experiments were stronger in the $y c g F^{+/-}$experiment at $16^{\circ} \mathrm{C}$ with blue-light irradiation, indicating that these conditions are the natural ones for strong activation of these genes.

Monitoring the effect of blue-light irradiation on growth rate at several temperatures revealed that blue light can be detrimental for growth specifically at very low temperature $\left(<23^{\circ} \mathrm{C}\right)$ (Supplemental Fig. S5). However, survival assays (i.e., the determination of colonyforming units) showed that blue-light irradiation at $16^{\circ} \mathrm{C}$ is not lethal for E. coli cells (Supplemental Fig. S6). The $y c g F$ mutation did not further affect growth rate or survival, indicating that while the $\mathrm{YcgF} / \mathrm{YcgE}$ system is activated by blue light in a cold environment, its downstream function is not to counteract any direct detrimental effects of blue light irradiation.

The YcgF/YcgE regulatory pathway affects biofilm and stress response functions via the Rcs phosphorelay system

What are the molecular and physiological functions of the eight small proteins under YcgF/YcgE control? When we cloned the $y c g Z$-ymg $A B$ region on a standard multicopy plasmid we noticed that transformants were mucoid; i.e., they produced more capsule material. In order to study this effect in more detail, we cloned all four genes separately as well as the entire operon under the control of the IPTG-inducible tac promoter $\left(t a c_{\mathrm{p}}\right)$ on a low-copynumber plasmid (pCAB18, which carries the p15A origin). We found that even with basal levels of expression (no inducer added), YmgB increased the production of capsule material already at $28^{\circ} \mathrm{C}$. At $16^{\circ} \mathrm{C}$, not only $\mathrm{YmgB}$, but also YmgA produced such an effect (Fig. 6A). At $16^{\circ} \mathrm{C}$, also the wild-type strain carrying the vector only had a moderate mucoid appearance that seemed to be less pronounced with the $y c g Z$-carrying plasmid, suggesting that YcgZ may have an effect opposite to that of YmgA/ YmgB. Mucoidy was due to the extracellular polysaccharide colanic acid, as it was abolished in a genetic background defective for the response regulator RcsB (Fig. 6A), which is essential for the expression of the enzymes that synthesize this biofilm matrix component (Majdalani and Gottesman 2005). That capsule genes were not significantly differentially regulated in our $y c g E^{+/-}$and $y c g F^{+/-}$ microarray experiments (Table 1; Supplemental Table S1) is probably due to the requirement of RcsA (besides RcsB) and surface growth conditions (as in Fig. 6A) for their expression (Majdalani and Gottesman 2005).

In addition, low-copy-number plasmid-encoded ymg $B$ interfered with the expression of the structural genes for curli fimbriae (as tested with the single-copy $\operatorname{csg} B:: 1 a c Z$ fusion at the standard temperature of curli expression; i.e., $28^{\circ} \mathrm{C}$ ) (Fig. $6 \mathrm{~B}$ ). At $16^{\circ} \mathrm{C}$, not only YmgB but also YmgA strongly reduced curli expression. At $28^{\circ} \mathrm{C}$, the presence of YcgZ somewhat alleviated the effect of YmgA/YmgB (when all proteins were expressed together from a single 


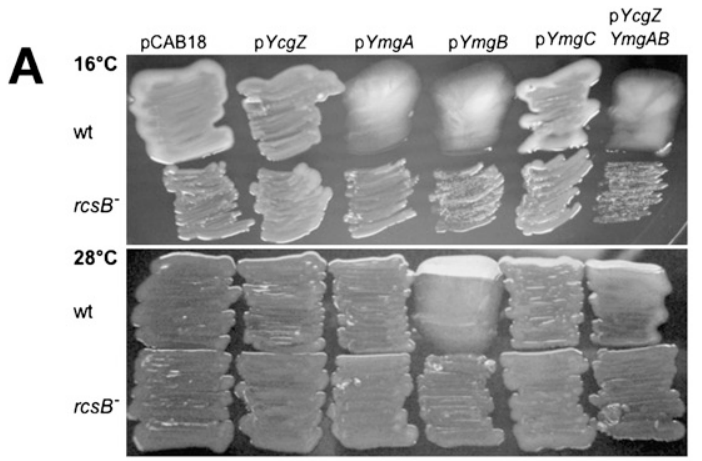

B
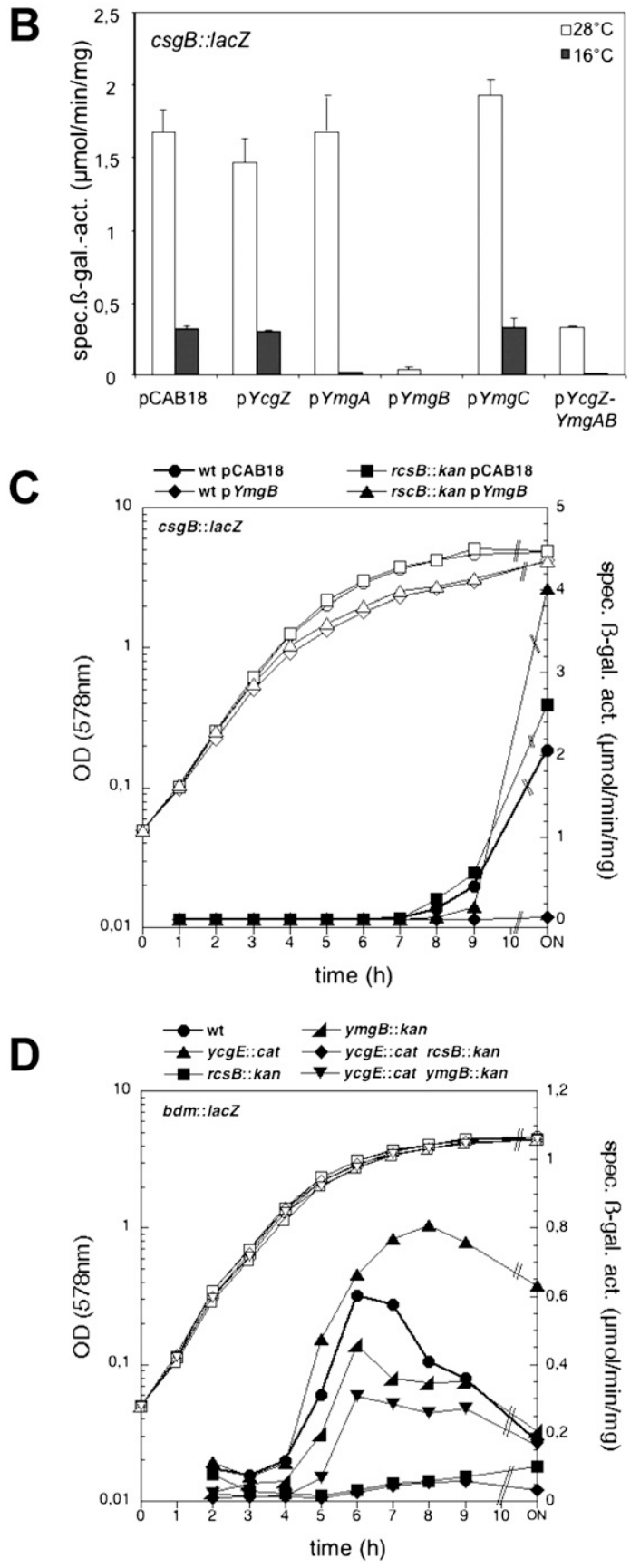

plasmid). Down-regulation of curli expression was also dependent on the presence of RcsB (Fig. 6C). Moreover, the presence of the $y m g B$-encoding plasmid stimulated the expression of the acid resistance gene gadB twofold to threefold during entry into stationary phase in an RcsB-dependent manner (assayed with a single-copy $\operatorname{gadB}:$ :lacZ fusion) (data not shown). This is consistent with $\operatorname{gadB}$ showing up as a moderately YcgE-repressed gene in our initial microarray analysis (Supplemental Table S1). Also, microarray-based transcriptome analysis (our unpublished data) confirmed that the presence of pYmgB strongly reduced the expression of the curli csg genes and activated numerous acid resistance genes as well as the small protein genes $b d m$ and $y b g S$. Moreover, among the additional genes affected, many were previously identified as RcsB-controlled (Hagiwara et al. 2003), but the $r c s C, r c s D$, and $r c s B$ genes themselves were not differentially regulated (data not shown).

Taken together, these data indicate that induction of YmgB modulates the activity (but not expression) of the Rcs two-component signaling pathway, that YmgA is a back-up component with similar activity that further boosts the response at very low temperature, and that YcgZ may partially (or conditionally) counteract the effects of YmgA/YmgB. Thus, the Rcs phosphorelay system with its multiple outputs seems a major indirect target of the $\mathrm{YcgF} / \mathrm{YcgE}$ pathway. In order to further corroborate this conclusion, we wanted to see whether such effects are elicited not only by YmgA/YmgB expressed from a plasmid, but also by the knockout mutation of $y c g E$ (the phenotype of which corresponds to the effect of full activation of YcgF by blue light) (see Fig. 5B). As a downstream target, we tested the expression of $b d m$ (as a single-copy lac $Z$ reporter fusion), as this gene is known to be under direct control of RcsB, which binds in the $b d m$ promoter region and does not require RcsA for activation (Francez-Charlot et al. 2005). Consistent with the microarray data (Table 1; Supplemental Table S1), the

Figure 6. Physiological effects of the small proteins induced by the $\mathrm{YcgF} / \mathrm{YcgE}$ signaling pathway. (A) Effects of the small proteins YcgZ, YmgA, YmgB, and YmgC on colony phenotype. MC4100 and its $r$ csB::kan derivative were transformed with pCAB18-derived plasmids expressing the small proteins as indicated and grown on $\mathrm{LB} /$ ampicilline plates at $16^{\circ} \mathrm{C}(50 \mathrm{~h})$ or $28^{\circ} \mathrm{C}(24 \mathrm{~h})$. (B) Effects of the small proteins YcgZ, YmgA, YmgB, and $\mathrm{YmgC}$ on curli fimbriae expression. MC4100 carrying $\operatorname{csg} B:: 1 a c Z$ fusion as well as the pCAB18-derived plasmids expressing the small proteins as indicated were grown in $\mathrm{LB} /$ ampicilline at $16^{\circ} \mathrm{C}$ and $28^{\circ} \mathrm{C}(50$ and $24 \mathrm{~h}$, respectively), and specific $\beta$-galactosidase activities were determined. $(C)$ Inhibition of curli expression by YmgB requires RcsB. MC4100 carrying $\operatorname{csg} B:: 1 a c Z$ and its $r \operatorname{cs} B::$ kan derivative also containing pYmgB or the vector pCAB18 were used. (D) A ycgE mutation affects $b d m$ expression via $\mathrm{YmgB}$ and RcsB. MC4100 carrying $b d m:: 1 a c Z$ and its derivatives with single or double mutations in $y \operatorname{cg} E, y m g B$, and $r c s B$ as indicated were used (note that the ymg $B:$ kan insertion shown as well as the nonpolar $y m g B$ flipout deletion showed the same effect). In $C$ and $D$, cells were grown in $\mathrm{LB} /$ ampicilline at $28^{\circ} \mathrm{C}$ and $\mathrm{OD}_{578}$ (open symbols), and specific $\beta$-galactosidase activities (closed symbols) were determined along the growth curve. 
ycgE mutation enhanced $b d m:: 1 a c Z$ expression, and this effect required the presence of RcsB (i.e., $r \operatorname{cs} B$ single and $r c s B$ ycgE double knockout mutant showed the same low expression of $b d m$; Fig. 6D). In addition, mutation of ymg $B$ slightly reduced $b d m:: 1 a c Z$ expression, and also in the $y m g B$-negative background, the $y c g E$ mutation did not enhance $b d m:: 1 a c Z$ expression (Fig. 6D); in fact, $b d m$ expression was even slightly reduced, consistent with higher expression of $\mathrm{YcgZ}$ in the $y c g E$ ymgB double mutant than in the $y m g B$ mutant. We conclude that also with wild-type expression levels of all components involved, the $\mathrm{YcgF} / \mathrm{YcgE}$ system acts via $\mathrm{YmgB}$ and the Rcs phosphorelay system on downstream targets.

\section{Discussion}

The EAL protein YcgF is not involved in c-di-GMP metabolism but acts by direct protein-protein interaction with the MerR-like repressor YcgE

As GGDEF and EAL domains have been associated with DGC and c-di-GMP phosphodiesterase activities, respectively, proteins carrying these domains are often assumed to be such enzymes without further testing. However, many bacteria have proteins in which the GGDEF and EAL motifs are degenerate, and as these amino acid motifs crucially contribute to the active centers of the enzymes (Malone et al. 2007; Wassmann et al. 2007; Rao et al. 2008), the degenerate proteins cannot have enzymatic activity. Nevertheless, degenerate GGDEF/EAL domain proteins can control various output functions, as shown for YhdA in E. coli (Suzuki et al. 2006; Sommerfeldt et al. 2009) and GdpS in Staphylococci (Holland et al. 2008). However, the direct molecular mechanisms of action of these proteins have remained unknown.

In the present study, we analyzed the function of a degenerate EAL protein in E. coli, YcgF, which also carries a blue-light-sensing BLUF domain at its $\mathrm{N}$ terminus. We found that $\mathrm{YcgF}$ acts by protein-protein interaction independently of c-di-GMP biochemistry. Purified YcgF did not degrade or bind c-di-GMP (Supplemental Fig. S1), nor did overproduction of an active DGC (YaiC) or an active PDE (YhjH) affect the expression of the YcgFcontrolled target gene ycgZ (Supplemental Fig. S2). Instead, YcgF directly interacts with the MerR-like repressor YcgE (Fig. 2) and, in a blue-light-dependent manner, releases it from the DNA, where YcgE specifically binds to the $y c g Z$ promoter region (Fig. 3; Supplemental Fig. S3). Thus, YcgF is a blue-light-controlled direct antirepressor for YcgE (for a summarizing model, see Fig. 7).

As both YcgF and YcgE are two-domain proteins, we studied the role of the specific domains in this interaction. The N-terminal BLUF domain of YcgF alone can be purified as a soluble protein, but it is unable to interact with YcgE. We conclude that the C-terminal EAL domain of YcgF is involved in this interaction, either on its own or in conjunction with the BLUF domain. Consistently, not only the BLUF domain but the entire YcgF protein changes conformation upon blue-light irradiation (Hasegawa et al. 2006; Nakasone et al. 2007). On the YcgE side, it is

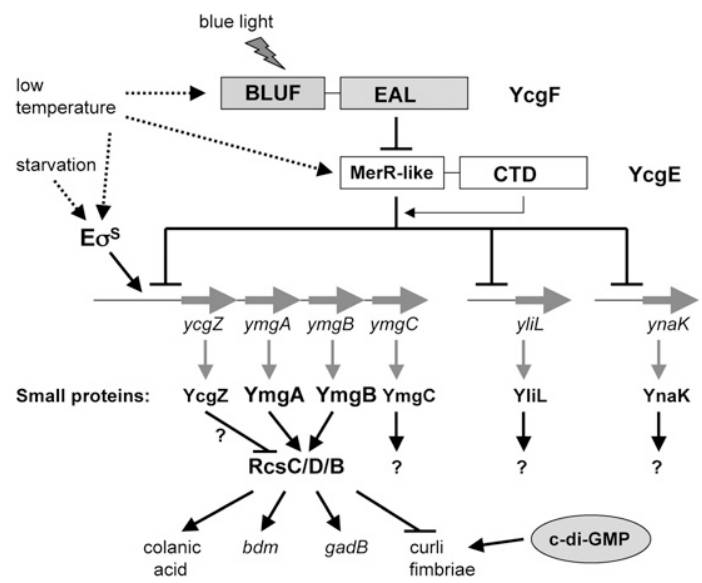

Figure 7. Model for the YcgF/YcgE-controlled small regulatory protein network. At the top of the network, YcgF acts as a direct antagonist for the MerR-like regulator YcgE. The roles of the Nand C-terminal domains of $\mathrm{YcgF}$ and YcgE are indicated (for details, see the Discussion). Inactivation of YcgE results in the induction of eight small proteins (YcgZ, YmgA, YmgB, YmgC, YliL, YnaK, Bdm, and YbgS; all between 71 and 126 amino acids long). YcgE directly represses the $y c g Z$-ymgABC operon and down-regulates also yliL and ynaK by yet uncharacterized mechanisms. Among the small proteins induced, YmgA and YmgB activate the Rcs phosphorelay pathway, and YcgZ has a weak opposite effect. Expression of the small protein genes $b d m$ and $y b g S$ (but not yliL and ynaK) is activated by YmgB. The $b d m$ gene is a known member of the RcsB regulon; how YmgB affects the $y b g S$ gene has yet to be clarified (therefore, $y b g S$ is not included in the figure). Several signals are integrated by the YcgF/YcgE pathway: Blue light affects the activity of YcgF protein; low temperature induces the expression of $\mathrm{YcgF}$, YcgE, and $\sigma^{\mathrm{S}}$; and starvation (and other stresses) results in $\sigma^{\mathrm{S}}$ accumulation due to post-transcriptional control mechanisms. For further details and references, see the Discussion.

the MerR-like DNA-binding N-terminal domain that binds to YcgF (Fig. 2). The molecular role of the YcgECTD is currently unknown, but it seems to contribute to repression as (1) expression of the DNA-binding YcgENTD alone is not sufficient to achieve complementation of a $y c g E$ mutation (assayed as repression of $y c g Z:: 1 a c Z$ ), and (2) expression of YcgE from a leaky overexpression vector resulted in repeatedly picking up inactivating point mutations not only in the DNA-binding helix of YcgE-NTD, but also at a specific position in the C-terminal domain (our unpublished results).

Here, we show that blue light is required for YcgF to release $\mathrm{YcgE}$ from its operator in the $y c g Z$ promoter region in vitro (Fig. 3). In vivo, blue light further increased YcgF-mediated activation of $y c g Z$ expression at low temperature (Fig. 5). Interestingly, also nonirradiated YcgF can bind to free YcgE (no DNA present) (Fig. 2). However, only irradiated YcgF can efficiently release YcgE bound to its operator DNA (Fig. 3). In principle, two different mechanisms could account for this regulation by blue light: Either YcgF binds equally well to free and DNA-bound YcgE, and the blue-light-induced conformational change in YcgF induces a conformational change in 
YcgE followed by release from the DNA; or, alternatively, YcgF may bind better to free YcgE as YcgF has to compete with operator DNA for overlapping binding sites in the MerR-like domain of YcgE, and blue light would increase the affinity of YcgF for YcgE such that it competes better with operator DNA. Several observations seem to support the second model. First, increased YcgF:YcgE stoichiometry can clearly induce target gene expression in vivo (overexpression of YcgF from a plasmid as in Fig. $1 \mathrm{C}$, or stronger induction of YcgF by low temperature as in Fig. 5; see also below); this is a key prediction of the competition model, but does not seem consistent with the first model. Also, the first model, in which YcgF binding does not discriminate between free and DNAbound YcgE, seems at odds with the finding that the order of addition of components and blue-light irradiation clearly affects the efficiency of release of YcgE from the DNA (Fig. 3). In conclusion, the current data indicate that blue-light irradiation increases $\mathrm{YcgF}$ affinity for $\mathrm{YcgE}$, thereby allowing it to release YcgE from its operator site, but this will have to be confirmed by further biochemical and structural analyses.

A similar situation as in the $\mathrm{YcgF} / \mathrm{YcgE}$ system has been described for CarS, which is a direct antagonist for the MerR-like regulator CarA that controls carotenoid biosynthesis in Myxococcus xanthus. The acidic CarS protein (theoretical pI of 4.8) structurally mimics operator DNA and thereby occludes the DNA-binding site in CarA (Navarro-Avilés et al. 2007). The theoretical pI of the YcgF-EAL domain is also acidic (4.82), which is in contrast to the more consensus-like YcgF-EAL domains of two bacterial species that do not have YcgE (5.29 and 5.28 in Bordetella avium and Alteromonas macleodii, respectively) (see Supplemental Fig. S1) and to the EAL domain of known PDE (e.g., YciR, pI of 6.03).

It should also be noted that the only paralog of YcgE in E. coli, which contains both a MerR-like NTD as well as a YcgE-like CTD, is MlrA. In contrast to the repressor YcgE, this protein acts as an activator that is essential and highly specific for the transcription of the curli activator gene $\operatorname{csg} D$ (Brown et al. 2001). The c-di-GMP phosphodiesterase YciR equally selectively inhibits $c s g D$ transcription (Weber et al. 2006; Pesavento et al. 2008). Preliminary data suggest that YciR (a GGDEF+EAL protein) directly interacts with MlrA and that this interaction is important for inhibiting $\operatorname{csg} D$ expression (S. Lindenberg, C. Pesavento, and R. Hengge, unpubl.). Thus, control of the activity of regulators by physical interaction with an EAL domain protein may be a more general principle and may be one way to achieve a more local action of GGDEF/EAL proteins, as suggested by several investigators (Jenal and Malone 2006; Kader et al. 2006; Ryan et al. 2006; Weber et al. 2006; Pesavento et al. 2008). C-di-GMP production and degradation may still play a role in such a complex (as it does for curli regulation; Weber et al. 2006). Alternatively, as in the case of $\mathrm{YcgF} / \mathrm{YcgE}$, it may have been "given up" during the course of evolution (see Supplemental Fig. S1), with the system now relying completely on protein-protein interaction.
Integration of blue light, low temperature, and general stress signals in the $Y c g F / Y c g E$ signaling pathway

The expression of both $y c g F$ and $y c g E$ as well as their target operon ycgZ-ymgABC is clearly cold-induced (Figs. 4, 5; Polissi et al. 2003; White-Ziegler et al. 2008). While the $y c g Z$-ymgABC operon is under $\sigma^{\mathrm{S}}$ control (Table 1; Weber et al. 2005), and therefore cold induction of $\sigma^{\mathrm{S}}$ itself (Sledjeski et al. 1996) may contribute to up-regulation of these genes, $y c g F$ and $y c g E$ expression is independent of $\sigma^{\mathrm{S}}$, and cold induction occurs by a currently unknown mechanism. Our data indicate that a shift to low temperature $\left(16^{\circ} \mathrm{C}\right)$ alone can already trigger a YcgF-mediated relief of repression by YcgE, which is further enhanced by blue-light irradiation (Fig. 5). We noted that low temperature induction of $y c g F$ expression is clearly stronger than that of $y \operatorname{cg} E(\sim 30$-fold and sevenfold, respectively, when cells were shifted from $37^{\circ} \mathrm{C}$ to $16^{\circ} \mathrm{C}$ ). Blue light promotes a conformational change of YcgF (Hasegawa et al. 2006; Nakasone et al. 2007; Schroeder et al. 2008) that probably increases YcgF affinity for YcgE and allows it to release YcgE from its operator site (as discussed above). Yet, by simple mass action, the cold-induced increase in the YcgF:YcgE stoichiometry also could have similar consequences; i.e., generate enough active YcgF to sequester $\mathrm{YcgE}$ and therefore induce the YcgE regulon. Interestingly, the recovery of the dark ground state of YcgF also is strongly delayed at lower temperature (Schroeder et al. 2008). Therefore, blue light and low temperature can act in an additive manner on target gene expression.

In addition, the general stress response feeds into in this complex regulatory circuit. Five of the eight target genes of the $\mathrm{YcgF} / \mathrm{YcgE}$ system require $\sigma^{\mathrm{S}}$ for expression (Table 1). $\sigma^{\mathrm{S}}$ accumulates under a variety of stress conditions (nutrient limitation, hyperosmotic, acidic, and other conditions) (Hengge-Aronis 2002). Given the combination of nutrient scarcity, low temperature, and intensive bluelight irradiation as inducing conditions, it is tempting to speculate that the YcgF/YcgE pathway is important for $E$. coli growth and/or survival in a marine or freshwater environment.

\section{The YcgF/YcgE pathway controls the expression of small regulatory proteins that modulate biofilm functions via the Rcs two-component pathway}

Is blue light just a signal indicative of an aquatic environment, or can blue light also be harmful on its own; i.e., cause cellular damage? We observed that blue-light irradiation indeed reduces growth of $E$. coli specifically at low temperature $\left(<23^{\circ} \mathrm{C}\right)$, but does not lead to increased cell death (Supplemental Figs. S5, S6). It could be that some particularly sensitive target for blue-light-induced photo-oxidation (Rajagopal et al. 2004) is formed at low temperature only (e.g., cold-adapted phospholipids with unsaturated fatty acid side chains and other modifications; Cronan 2003). However, mutants defective for YcgF, $\mathrm{RcsB}$, or even $\sigma^{\mathrm{S}}$ do not show further reduced growth or even cell death upon blue-light irradiation (Supplemental Fig. S6), indicating that the $\mathrm{YcgF} / \mathrm{YcgE}$ pathway does not 
cope with blue-light/cold-induced cellular stress or damage, but uses blue light and low temperature as distinct environmental indicator signals.

What are the output functions of the $\mathrm{YcgF} / \mathrm{YcgE}$ pathway, and how are these of adaptive value in a nutrient-limited, cold, and radiation-rich environment? The primary output of this pathway is the expression of six small proteins (Fig. 7). Four are encoded in an operon, $y c g Z$-ymgABC, which is under direct control of the YcgE repressor. It is not yet clear whether the target genes yliL and $y n a K$ are under similar direct control, but they are at least not activated by the primary target $\mathrm{YmgB}$, as are two additional genes, $b d m$ and $y b g S$, which therefore are indirect downstream targets of the $\mathrm{YcgF} / \mathrm{YcgE}$ pathway (Fig. 6D; our unpublished microarray data). This distinct group of eight coregulated small proteins (78-126 amino acids) is so far unique.

We showed here that $\mathrm{YmgB}$, and to some extent also YmgA (in particular at $16^{\circ} \mathrm{C}$ ), can strongly promote the synthesis of the exopolysaccharide and biofilm matrix component colanic acid and virtually eliminates the expression of the adhesive curli fimbriae genes, whereas YcgZ seemed to partially antagonize these effects (Fig. 6). In addition, YmgB moderately stimulated the expression of several acid resistance genes including gadB, gadC, gadE, and hdeA (data not shown). These findings are consistent with a recent report showing that YmgB reduces biofilm formation (tested only in the "crystal violett assay," which, however, is known to be sensitive to curli expression) and promotes survival at low $\mathrm{pH}$ (Lee et al. 2007). Moreover, all YmgB-regulated functions that we describe here-i.e., colanic acid production, curli biosynthesis, and the expression of acid resistance genes, $b d m$ and many other genes-are under direct or indirect control of the $\mathrm{R} c s \mathrm{C} / \mathrm{R} \operatorname{csD} / \mathrm{R} \operatorname{csB}$ two-component phosphorelay system (Gottesman et al. 1985; Hagiwara et al. 2003; Francez-Charlot et al. 2005; Vianney et al. 2005; Castanié-Cornet et al. 2007). As these YmgB effects were eliminated in an $r c s B$ mutant background (Fig. 6; data not shown), we conclude that $\mathrm{YmgB}$ acts upstream of and via RcsB (Fig. 7). Moreover, YmgB stimulates the activity rather than the expression of the Rcs system, as $r c s B$, $r c s C$, and $r c s D$ were not among the genes activated by YmgB. Using the known RcsB-targeted $b d m$ gene as a reporter in an epistasis analysis, we show that also a knockout mutation in $y c g E$ affects $b d m$ expression via YmgB and RcsB (Fig. 6D). That the $\mathrm{YcgF} / \mathrm{YcgE} / \mathrm{YmgB}$ pathway can activate the Rcs system is consistent with the $y c g Z$-ymgABC genes belonging to a cohort of genes that exhibit intermediate timing of expression during biofilm development (Domka et al. 2007), which fits the concept that the Rcs system is involved in biofilm maturation rather than initiation (Majdalani and Gottesman 2005; Beloin et al. 2008). Thus, via YmgB/YmgA and $\mathrm{RcsB}$, the $\mathrm{YcgF} / \mathrm{YcgE}$ system can stimulate biofilm maturation and possibly differential gene expression within a biofilm. In a natural aquatic environment, a thick biofilm matrix may not only afford protection against abiotic stresses, but, e.g., also against grazing microbial predators.
What is the molecular mechanism of action of $\mathrm{YmgB}$, $\mathrm{YmgA}$, and the other small proteins under $\mathrm{YcgF} / \mathrm{YcgE}$ control? As YmgB is a small three-helix protein that exhibits similarity to Hha, a protein that interacts with the DNA-binding protein $\mathrm{H}-\mathrm{NS}$, it was speculated that YmgB might be a novel type of transcriptional (co)regulator (Lee et al. 2007). As YmgB clearly acts upstream of and via RcsB (see above), we propose the hypothesis that YmgB (and perhaps also YmgA) could be a two-component system "connector" that specifically activates the Rcs phosphorelay system. Connectors are a currently emerging group of typically small proteins $(\sim 60-150$ amino acids) that influence the activity of specific twocomponent systems. They provide additional signal input not perceived by the sensory input domains of the sensor kinases, and thereby connect two-component systems to other regulatory pathways. Connectors can affect phosphorylation, phosphotransfer, and dephosphorylation reactions within simple two-component or more complex phosphorelay systems or they may sequester a component of such a pathway (Kato and Groisman 2004; Bougdour et al. 2006, 2008; Eguchi et al. 2007; Kato et al. 2007). How YmgB and probably also YmgA and YcgZ affect the activity of the Rcs phosphorelay will have to be investigated in future genetic and biochemical studies. Furthermore, it is conceivable that other small proteins under the control of the $\mathrm{YcgF} / \mathrm{YcgE}$ system might also act as connectors. In this case, the $\mathrm{YcgF} / \mathrm{YcgE}$ system would not only modulate biofilm formation, but could reorganize the two-component network, when $E$. coli has to survive in a marine or freshwater environment characterized by starvation, cold, and sun radiation.

\section{Materials and methods}

Bacterial strains and growth conditions

All strains used in this study are derivatives of the E. coli K-12 strain MC4100 (Casadaban 1976). Mutations were introduced by P1 transduction (Miller 1972). The rpoS359::Tn10 mutation was described previously (Lange and Hengge-Aronis 1991). The newly constructed mutant alleles ycgF::kan, ycgE::cat, $\Delta(y c g Z$ ymg $A B):: c a t, y m g B:: k a n$, and $r c s B:: k a n$ are all deletion-insertion mutations generated by one-step inactivation according to Datsenko and Wanner (2000) using the primers listed in Supplemental Table S2. In order to avoid polarity on downstream genes in an operon, insertions were also flipped out according to Datsenko and Wanner (2000). The construction of plasmids and single-copy lacZ reporter fusions is described in detail in the Supplemental Material (including relevant references). Note that $y c g F$ and $y c g E$ do not constitute an operon (for experimental evidence, see Supplemental Material). Thus, the ycgF insertion is also not polar on $y c g E$.

Cells were grown in LB medium (Miller 1972) under aeration at the temperatures indicated in the figure legends. Antibiotics and IPTG were added as recommended (Miller 1972). Growth was monitored by measuring the optical density at $578 \mathrm{~nm}$ $\left(\mathrm{OD}_{578}\right)$. Blue-light $(470 \mathrm{~nm})$ irradiation of growing bacterial cultures or protein samples was performed with an intensity of $\sim 100 \mu \mathrm{M} / \mathrm{m}^{-2} \mathrm{sec}^{-1}$ using 112 5-mm LEDs (HLMP-HB57-LP0xx, Avago Technologies) arranged as a $16 \times 7$ array on a $16 \times 10$-cm plate. Survival of blue-light exposure during growth was monitored 
by determining colony forming units (on LB plates) in samples taken from the irradiated cultures.

\section{Northern blot analysis}

RNA was prepared and Northern blot analysis was performed as described before (Weber et al. 2006) using a DIG-labeled probe corresponding to the $y c g Z$ coding region (obtained with primers listed in Supplemental Table S2).

\section{Microarray analysis}

Cell lysis, RNA isolation, DNaseI treatment, and phenol/chloroform extraction were previously described (Weber et al. 2006). E. coli K-12 microarrays containing 4288 gene-specific $50 \mathrm{mer}$ oligonucleotide probes representing the whole E. coli genome (MWG) were used for hybridization with Cy3/5-dCTP-labeled cDNA as described in the supplemental material of Pesavento et al. (2008). Fluorescence detection and image analysis was carried out as described before (Weber et al. 2005) using a GenePix 4100A (Axon, Inc.) laser scanner. Each microarray experiment was repeated two times (biological replicates, with a dye swap in cDNA labeling). Genes were considered differentially regulated when (1) signal-to-noise ratios exceeded a factor of three, (2) the sum of median intensity counts was $>200$, and (3) relative RNA level differences (ratios) were at least twofold in both of the two independent experiments. The original data sets can be found in the Array Express database (accession no. E-MEXP-1872 for the $\mathrm{YcgE}^{+/-}$data, E-MEXP-1877 for the $\mathrm{YcgF}^{+/-}$data)

\section{In vitro protein-protein interaction assay and SDS-PAGE}

Purification of YcgF and YcgE and their respective domains with TRX-His6-S and/or His6 tags is described in detail in the Supplemental Material. In vitro interaction assays were performed by affinity chromatography ("pull-down" assays) on Sprotein agarose using $0.65 \mathrm{nmol}$ of YcgE or the YcgE domains and $1.3 \mathrm{nmol}$ of YcgF or YcgF-NTD. The procedure was as described by Mika and Hengge (2005), with the exception that the samples were washed five times instead of twice only, and elution was with $3 \mathrm{M} \mathrm{MgCl}_{2}$. Eluates were analyzed by $12 \%$ SDS-PAGE and proteins were detected by Coomassie brilliant blue staining.

\section{Protein-DNA interaction assays}

Electrophoretic mobility shift assays (EMSA) were essentially performed as described (Mika and Hengge 2005). DNA fragments used carried either the $y c g Z$ promoter or the $y c g Z$-coding regions and were obtained with the primers listed in Supplemental Table S2. Twenty-microliter reaction mixtures contained purified YcgE protein $(0.47 \mu \mathrm{M})$ and $18 \mathrm{ng}$ of the DNA fragments in $10 \mathrm{mM}$ Tris$\mathrm{HCl}(\mathrm{pH} 7.5), 10 \mathrm{mM} \mathrm{NaCl}, 1 \mathrm{mM} \mathrm{MgCl} 2,1 \mathrm{mM}$ EDTA, $5 \mathrm{mM}$ DTT, and 5\% glycerol. YcgE, YcgF, and DNA fragments were added in the order and concentrations stated in the text and the figure legends (at room temperature). Preincubation of two components was for $20 \mathrm{~min}$ before the third component was added. Blue-light irradiation was also for $20 \mathrm{~min}$ (with control samples left in the dark in parallel). Samples were then run on a $1 \%$ agarose gel in TAE buffer and stained with ethidium bromide.

Determination of c-di-GMP binding and phosphodiesterase activity

In vitro synthesis of radiolabeled c-di-GMP by the purified DGC $\mathrm{PleD}^{\star}$, and purification of c-di-GMP was done as described (Paul et al. 2004; Weber et al. 2006). Binding of radiolabeled c-di-GMP to purified proteins in vitro was detected by UV cross-linking according to Christen et al. (2007). Phosphodiesterase activity was tested with purified $\mathrm{YcgF}$ and $\mathrm{YcgF}^{\mathrm{I193L} / \mathrm{Q} 195 \mathrm{R}}$ (see the Supplemental Material for details) and C-terminally His6-tagged YhjH (Pesavento et al. 2008) using radiolabelled c-di-GMP as a substrate following the procedure described in detail by Weber et al. (2006).

\section{Determination of $\beta$-galactosidase activity}

$\beta$-galactosidase activity was assayed by use of $o$-nitrophenyl- $\beta$-Dgalactopyranoside (ONPG) as a substrate and is reported as micromoles of $o$-nitrophenol per minute per milligram of cellular protein (Miller 1972). Experiments showing the expression of lac $Z$ fusions along the entire growth cycle were done at least twice, and a representative experiment is shown. Single-value data are the average of at least two independent measurements.

\section{Acknowledgments}

Financial support was provided by the Deutsche Forschungsgemeinschaft (He 1556/13-1), the Dr. Hans-Messner-Stiftung, and the Fonds der Chemischen Industrie.

\section{References}

Beloin, C., Roux, A., and Ghigo, J.-M. 2008. Escherichia coli biofilms. Curr. Top. Microbiol. Immunol. 322: 249-289.

Bougdour, A., Wickner, S., and Gottesman, S. 2006. Modulating RssB activity: IraP, a novel regulator of $\sigma^{\mathrm{S}}$ stability in Escherichia coli. Genes \& Dev. 20: 884-897.

Bougdour, A., Cunning, C., Baptiste, P.J., Elliott, T., and Gottesman, S. 2008. Multiple pathways for regulation of $\sigma^{\mathrm{S}}\left(\mathrm{Rpo}^{\mathrm{S}}\right)$ stability in Escherichia coli via the action of multiple antiadaptors. Mol. Microbiol. 68: 298-313.

Brown, P.K., Dozois, C.M., Nickerson, C.A., Zuppardo, A., Terlonge, J., and Curtiss III., R. 2001. MlrA, a novel regulator of curli (Agf) and extracellular matrix synthesis by Escherichia coli and Salmonella enterica serovar typhimurium. Mol. Microbiol. 41: 349-363.

Casadaban, M.J. 1976. Transposition and fusion of the lac genes to selected promoters in Escherichia coli using bacteriophage $\lambda$ and Mu. J. Mol. Biol. 104: 541-555.

Castanié-Cornet, M.-P., Treffandier, H., Francez-Charlot, A., Gutierrez, C., and Cam, K. 2007. The glutamate-dependent acid resistance system in Escherichia coli: Essential and dual role of the His-Asp phosphorelay RcsCDB/AF. Microbiology 153: 238-246.

Christen, M., Christen, B., Allan, M.G., Folcher, M., Jenö, P., Grzesiek, S., and Jenal, U. 2007. DgrA is a member of a new family of cyclic diguanosine monophosphate receptors and controls flagellar motor function in Caulobacter crescentus. Proc. Natl. Acad. Sci. 104: 4112-4117.

Cotter, P.A. and Stibitz, S. 2007. c-di-GMP-mediated regulation of virulence and biofilm formation. Curr. Opin. Microbiol. 10: $17-23$.

Cronan, J.E. 2003. Bacterial membrane lipids: Where do we stand? Annu. Rev. Microbiol. 57: 203-224.

Datsenko, K.A. and Wanner, B.L. 2000. One-step inactivation of chromosomal genes in Escherichia coli K-12 using PCR products. Proc. Natl. Acad. Sci. 97: 6640-6645. 
Domka, J., Lee, J., Bansal, T., and Wood, T.K. 2007. Temporal gene expression in Escherichia coli K-12 biofilms. Environ. Microbiol. 9: 332-346.

Eguchi, Y., Itou, J., Yamane, M., Demizu, R., Yamato, F., Okada, A., Mori, H., Kato, A., and Utsumi, R. 2007. B1500, a small membrane protein, connects the two-component systems EvgS/EvgA and PhoQ/PhoP in Escherichia coli. Proc. Natl. Acad. Sci. 104: 18712-18717.

Francez-Charlot, A., Castanié-Cornet, M.-P., Gutierrez, C., and Cam, K. 2005. Osmotic regulation of the Escherichia coli bdm (biofilm-dependent modulation) gene by the RcsCDB His-Asp phosphorelay. J. Bacteriol. 187: 3873-3877.

Gomelsky, M. and Klug, G. 2002. BLUF: A novel FAD-binding domain involved in sensory transduction in microorganisms. Trends Biochem. Sci. 27: 497-500.

Gottesman, S., Trisler, P., and Torres-Cabassa, A.S. 1985. Regulation of capsular polysaccharide synthesis in Escherichia coli K12: Characterization of three regulatory genes. $J$. Bacteriol. 162: 1111-1119.

Hagiwara, D., Sugiura, M., Oshima, T., Mori, H., Aiba, H., Yamashino, T., and Mizuno, T. 2003. Genome-wide analyses revealing a signaling network of the RcsC-YojN-RcsB phosphorelay system in Escherichia coli. J. Bacteriol. 185: 5735-5746.

Hasegawa, K., Masuda, S., and Ono, T.A. 2006. Light induced structural changes of a full-length protein and its BLUF domain in YcgF (Blrp), a blue-light sensing protein that uses FAD (BLUF). Biochemistry 45: 3785-3793.

Hengge-Aronis, R. 2002. Signal transduction and regulatory mechanisms involved in control of the $\sigma^{\mathrm{S}}$ subunit of RNA polymerase in Escherichia coli. Microbiol. Mol. Biol. Rev. 66: 373-395.

Holland, L.M., O'Donnell, S.T., Ryjenkov, D.A., Gomelsky, L., Slater, S.R., Fey, P.D., Gomelsky, M., and O'Gara, J.P. 2008. A staphylococcal GGDEF domain protein regulates biofilm formation independently of c-di-GMP. I. Bacteriol. 190: 5178-5189.

Jenal, U. and Malone, J. 2006. Mechanisms of cyclic-di-GMP signaling in bacteria. Annu. Rev. Genet. 40: 385-407.

Kader, A., Simm, R., Gerstel, U., Morr, M., and Römling, U. 2006. Hierarchical involvement of various GGDEF domain proteins in rdar morphotype development of Salmonella enterica serovar typhimurium. Mol. Microbiol. 60: 602-616.

Kato, A. and Groisman, E.A. 2004. Connecting two-component regulatory systems by a protein that protects a response regulator from dephosphorylation by its cognate sensor. Genes \& Dev. 18: 2302-2313.

Kato, A., Mitrophanov, A.Y., and Groisman, E.A. 2007. A connector of two-component regulatory systems promotes signal amplification and persistence of expression. Proc. Natl. Acad. Sci. 104: 12063-12068.

Lange, R. and Hengge-Aronis, R. 1991. Identification of a central regulator of stationary-phase gene expression in Escherichia coli. Mol. Microbiol. 5: 49-59.

Lee, J., Page, R., García-Contreras, R., Palermino, J.-M., Zhang, X.-S., Doshi, O., Wood, T.K., and Peti, W. 2007. Structure and function of the Escherichia coli protein YmgB: A protein critical for biofilm formation and acid resistance. J. Mol. Biol. 373: $11-26$.

Majdalani, N. and Gottesman, S. 2005. The Rcs phosphorelay: A complex signal transduction system. Annu. Rev. Microbiol. 599: 379-405.

Malone, J., Williams, R., Christen, M., Jenal, U., Spiers, A.J., and Rainey, P.B. 2007. The structure-function relationship of WspR, a Pseudomonas fluorescens response regulator with a GGDEF output domain. Microbiology 153: 980-994.
Mika, F. and Hengge, R. 2005. A two-component phosphotransfer network involving ArcB, ArcA and RssB coordinates synthesis and proteolysis of $\sigma^{\mathrm{s}}$ in E. coli. Genes \& Dev. 19: 2770-2781.

Miller, J.H. 1972. Experiments in molecular genetics. Cold Spring Harbor Laboratory, Cold Spring Harbor, NY.

Nakasone, Y., Ono, T.A., Ishii, A., Masuda, S., and Terazima, M. 2007. Transient dimerization and conformational change of a BLUF protein: YcgF. J. Am. Chem. Soc. 129: 7028-7035.

Navarro-Avilés, G., Jiménez, M.A., Pérez-Marín, M., González, C., Rico, M., Murillo, F.J., Elías-Arnanz, M., and Pdmanabhan, S. 2007. Structural basis for operator and antirepressor recognition by Myxococcus xanthus CarA repressor. Mol. Microbiol. 63: 980-994.

Paul, R., Weiser, S., Amiot, N., Chan, C., Schirmer, T., Giese, B., and Jenal, U. 2004. Cell cycle-dependent dynamic localization of a bacterial response regulator with a novel diguanylate cyclase output domain. Genes \& Dev. 18: 715-727.

Pesavento, C., Becker, G., Sommerfeldt, N., Possling, A., Tschowri, N., Mehlis, A., and Hengge, R. 2008. Inverse regulatory coordination of motility and curli-mediated adhesion in Escherichia coli. Genes \& Dev. 22: 2434-2446.

Polissi, A., De Laurentis, W., Zangrossi, S., Briani, F., Longhi, V., Pesole, G., and Dehò, G. 2003. Changes in Escherichia coli transcriptome during acclimatization at low temperature. Res. Microbiol. 154: 573-580.

Purcell, E.B. and Crosson, S. 2008. Photoregulation in prokaryotes. Curr. Opin. Microbiol. 11: 168-178.

Rajagopal, S., Key, J.M., Purcell, E.B., Boerema, D.J., and Moffat, K. 2004. Purification and initial characterization of a putative blue light-regulated phosphodiesterase from Escherichia coli. Photochem. Photobiol. 80: 542-547.

Rao, F., Yang, Y., Qi, Y., and Liang, Z.X. 2008. Catalytic mechanism of c-di-GMP specific phosphodiesterase: A study of the EAL domain-containing RocR from Pseudomonas aeruginosa. J. Bacteriol. 190: 3622-3631.

Römling, U. and Amikam, D. 2006. Cyclic di-GMP as a second messenger. Curr. Opin. Microbiol. 9: 1-11.

Ryan, R.P., Fouhy, Y., Lucey, F., and Dow, J.M. 2006. Cyclic diGMP signaling in bacteria: Recent advances and new puzzles. J. Bacteriol. 188: 8327-8334.

Schroeder, C., Werner, K., Otten, H., Krätzig, S., Schwalbe, H., and Essen, L.-O. 2008. Influence of a joining helix on the BLUF domain of the YcgF photoreceptor from Escherichia coli. ChemBioChem 9: 2463-2473.

Sledjeski, D.D., Gupta, A., and Gottesman, S. 1996. The small RNA, DsrA, is essential for the low temperature expression of RpoS during exponential growth in E. coli. EMBO J. 15: 3993-4000.

Sommerfeldt, N., Possling, A., Becker, G., Pesavento, C., Tschowri, N., and Hengge, R. 2009. Gene expression patterns and differential input into curli fimbriae regulation of all GGDEF/EAL domain proteins in Escherichia coli. Microbiology (in press).

Suzuki, K., Babitzke, P., Kushner, S.R., and Romeo, T. 2006. Identification of a novel regulatory protein $(\mathrm{CsrD})$ that targets the global regulatory RNAs CsrB and CsrC for degradation by RNase E. Genes \& Dev. 20: 2605-2617.

Tamayo, R., Pratt, J.T., and Camilli, A. 2007. Roles of cyclic diguanylate in the regulation of bacterial pathogenesis. Annu. Rev. Microbiol. 61: 131-148.

Vianney, A., Jubelin, G., Renault, S., Dorel, C., Lejeune, P., and Lazzaroni, J.C. 2005. Escherichia coli tol and rcs genes participate in the complex network affecting curli synthesis. Microbiology 151: 2487-2497. 
Tschowri et al.

Wassmann, P., Chan, C., Paul, R., Beck, A., Heerklotz, H., Jenal, U., and Schirmer, T. 2007. Structure of BeF3-modified response regulator PleD: Implications of diguanylate cyclase activation, catalysis, and feedback inhibition. Structure 15: 915-927.

Weber, H., Polen, T., Heuveling, J., Wendisch, V., and Hengge, R. 2005. Genome-wide analysis of the general stress response network in Escherichia coli: $\sigma^{\mathrm{s}}$-dependent genes, promoters and $\sigma$ factor selectivity. J. Bacteriol. 187: 1591-1603.

Weber, H., Pesavento, C., Possling, A., Tischendorf, G., and Hengge, R. 2006. Cyclic-di-GMP-mediated signaling within the $\sigma^{\mathrm{S}}$ network of Escherichia coli. Mol. Microbiol. 62: 10141034.

White-Ziegler, C.A., Um, S., Pérez, N.M., Berns, A.L., Malhowski, A.J., and Young, S. 2008. Low temperature $\left(23^{\circ} \mathrm{C}\right)$ increases expression of biofilm-, cold-shock- and RpoS-dependent genes in Escherichia coli K-12. Microbiology 154: 148-166. 


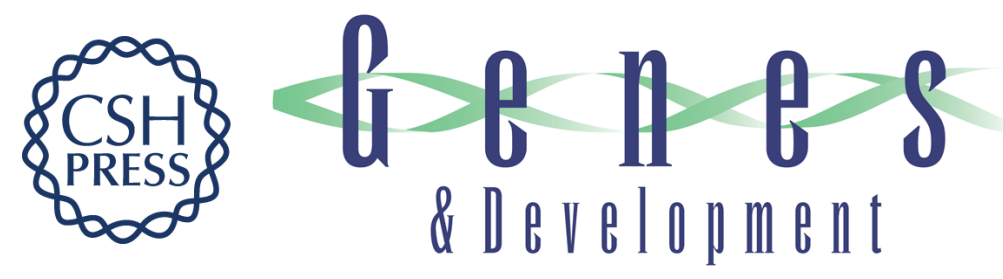

\section{The BLUF-EAL protein YcgF acts as a direct anti-repressor in a blue-light response of Escherichia coli}

Natalia Tschowri, Susan Busse and Regine Hengge

Genes Dev. 2009, 23:

Access the most recent version at doi:10.1101/gad.499409

Supplemental http://genesdev.cshlp.org/content/suppl/2009/02/24/23.4.522.DC1
Material

References This article cites 47 articles, 18 of which can be accessed free at: http://genesdev.cshlp.org/content/23/4/522.full.html\#ref-list-1

License

Email Alerting Receive free email alerts when new articles cite this article - sign up in the box at the top Service right corner of the article or click here.

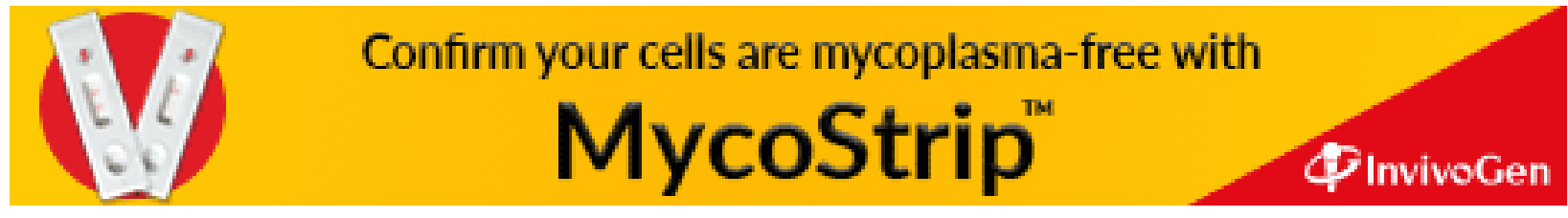

